ity and one common mechanism of its toxic action in different organs.

Methods: Bifenthrin at the doses of $8 \mathrm{mg} / \mathrm{kg}$ or $4 \mathrm{mg} / \mathrm{kg}$ of body mass was administered intraperitoneally to the mice for 28 subsequent days. Controls were injected with respective doses of saline. Motor function was measured on day 1, 7, 14 and 28 and memory retention was tested in a passive avoidance task on day 2 , 7, 14 and 28. On day 29 animals were decapitated, blood and livers were obtained for laboratory analyses. Blood morphology, alanine transaminase in the blood sera, superoxide dismuthase and glutathione peroxidase activities in the livers and microscopic examination of the livers were done.

Results: Bifenthrin significantly impaired memory retention on day 2 , decreased locomotor activity at every stage of the experiment in a single dose depending manner. No behavioral cumulative effect was observed. Subacute poisoning with the higher dose of bifenthrin caused anemia, elevated white blood cell count, elevated alanine transaminase, superoxide dismuthase, and decreased glutathione peroxidase activity. Lymphocyte infiltrates were visualized in the livers.

Conclusion: Subacute poisoning with bifenthrin decreases locomotor activity in a single dose proportionate manner. Bifenthrin damaged mice livers and altered blood morphology. The possible common mechanism of these effects is oxidative stress.

http://dx.doi.org/10.1016/j.toxlet.2015.08.794

\section{P13-006 \\ LmrBPP9: A synthetic bradykinin-potentiating peptide that presents hypotensive activity in hypertensive rats}

E. Pinheiro-Junior ${ }^{1}$,*, J. Boldrini-França ${ }^{1}$, L. Mattoso Pires de Campos Araújo $^{1}$, N. Alves Santos-Filho ${ }^{2}$, L. Maria Bendhack ${ }^{1}$, E. Maffud-Cilli $^{2}$, E. Candiani Arantes ${ }^{1}$

\section{${ }^{1}$ University of Sao Paulo, Physical Chemistry, Ribeirão Preto, Sao Paulo, Brazil \\ ${ }^{2}$ Chemical Institute of Araraquara - State University of Sao Paulo, Araraquara, Sao Paulo, Brazil}

Bradykinin-potentiating peptides (BPPs) are a major group of toxins present in Lachesis muta rhombeata venom. They act directly at renin-angiotensin-aldosterone system, through the inhibition of angiotensin-converting enzyme (ACE), responsible for producing the hypertensive peptide angiotensin II. This system plays a crucial role in the mechanisms that control the blood pressure, such as tubular salt reabsorption and retention of water in kidneys, aldosterone secretion and arteriolar vasoconstriction. Hypertension, a disease that can be triggered by a disruption in this system, is a leading cause of cardiovascular mortality. In this context, there is an interest in developing new drugs capable of controlling hypertension in humans, and one special target is the inhibition of ACE. Therefore, the main goal of this study was the solid-phase synthesis of a BPP found in Lachesis muta rhombeata venom and its characterization in vitro and in vivo in relation to ACE inhibition and hypotensive activity, respectively. The LmrBPP9 peptide was synthesized using an automated solid-phase peptide synthesizer and purified by reversed-phase fast protein liquid chromatography (RP-FPLC). The in vitro ACE inhibition assay was performed as previously described. The in vivo hypotensive effect of LmrBPP9 was evaluated in normotensive and renal hypertensive rats, using $2 \mathrm{~K}-$ 1C (two kidneys, one clip) model and three different concentrations of LmrBPP9. The in vitro $\mathrm{IC}_{50}$ of the synthetic peptide was $103 \mathrm{nM}$, showing a great capacity of ACE inhibition. The in vivo studies showed that LmrBPP9 induces a reduction in blood pressure, both in normotensive and hypertensive rats, being more pronounced in the last ones. These results are in agreement with the in vitro results, indicating that the synthetic peptide LmrBPP9 could be a potential molecule to the development of a new antihypertensive drug.

http://dx.doi.org/10.1016/j.toxlet.2015.08.795

\section{P13-007}

\section{Contribution of fipronil sulfone to the} fipronil-induced cytotoxicity

M.R. Martínez-Larrañaga*, A. Romero, I. Ares, E. Ramos, M. Martínez, V. Castellano, M. Martínez, A. Anadón

Universidad Complutense de Madrid, Department of Toxicology and Pharmacology, Faculty of Veterinary Medicine, Madrid, Spain

Fipronil is a pesticide of the phenylpyrazole family widely used as a phytosanitary product and as an insecticide in pets. Concerns for fipronil effects on public health have been raised because of the wide range of uses of this pesticide. Fipronil is moderately toxic to rats and mice with oral LD50's ranging from 40 to $100 \mathrm{mg} / \mathrm{kg}$, but is much more toxic toward insects than toward mammals. Fipronil selectivity is due to its greater potency in blocking insect GABA-gated chloride channels than their mammalian counterparts. Limited metabolic studies indicate that the predominant pathway of fipronil metabolism is S-oxidation to form the sulfone. Fipronil sulfone is the only metabolite reported in mice and in vitro studies suggest that fipronil sulfone is more potent as an antagonist of the GABA receptor than fipronil. Because metabolism may be an important determinant of toxicity, the present study was designed to compare the cytotoxicity of fipronil and its metabolite fipronil sulfone determining dose-response curves for cell viability MTT assay, LDH leakage, and malondialdehyde (MDA) and nitrite (NO) production as indicators of oxidative stress, a potential mechanism of fipronil toxicity. Human neuroblastoma SH-SY5Y cell line was selected as a sensitive in vitro model. Our results demonstrated that in neuroblastoma SH-SY5Y cells: (1) for MTT reduction and LDH leakage assays, fipronil ED50 values were greater than sulfone ED50 values; (2) both fipronil and its metabolite sulfone increased by 1.5 - and by 1.7 -fold NO production, respectively, and (3) both fipronil and its metabolite sulfone increased by 4.8 - and by 5.5 -fold MDA production, respectively. In conclusion, we evidenced that the cytotoxicity induced by fipronil sulfone was 50 times higher than the parental compound fipronil. Further investigations are required to determine the contribution of fipronil sulfone to mammalian toxicological effects. These findings underline the importance of characterizing the exposure to the toxicant and its metabolites in toxicological studies.

Acknowledgements: This work was supported by project UCMBSGH/920204 from Universidad Complutense de Madrid, and project(ALIBIRD-CM)S2013/ABI-2728 from Comunidad de Madrid.

http://dx.doi.org/10.1016/j.toxlet.2015.08.796 